\title{
Mechanical and electro-mechanical analysis in differently stabilized GdBCO coated conductor tapes with stainless steel substrate
}

\author{
Arman R. Nisay and Hyung-Seop Shin* \\ Department of Mechanical Design Engineering, Andong National University, Andong, Korea
}

(Received 28 May 2013; revised or reviewed 24 June 2013; accepted 25 June 2013)

\begin{abstract}
The understanding of the strain dependence of critical current, $I_{\mathrm{c}}$, in the reversible region is important for the evaluation of the performance of coated conductor (CC) tapes in practical applications. In this study, the stress/strain tolerance of $I_{\mathrm{c}}$ in GdBCO CC tapes with stainless steel substrate stabilized by additional $\mathrm{Cu}$ and brass laminate was analyzed quantitatively through $I_{\mathrm{c}}-$ strain measurement at $77 \mathrm{~K}$ under self-field. The variation in irreversible strain limits of CC tapes by the addition of stabilizing layers was analyzed through the consideration of the pre-strain induced on the GdBCO coating film. The results were then compared with the ones previously reported for GdBCO CC tapes with Hastelloy substrate. As a result, GdBCO CC tapes with stainless steel substrate showed much higher strain tolerance of $I_{\mathrm{c}}$ as compared with those adopting Hastelloy substrate.
\end{abstract}

Keywords: coated conductor, critical current, GdBCO, irreversible strain limit, stainless steel substrate, stabilizing layer, pre-strain

\section{INTRODUCTION}

REBCO coated conductor (CC) tapes have been recently used in practical applications such as power cables, rotating machines, coil windings, and magnets [1-6] since these CC tapes have shown superior superconducting properties at external magnetic fields and have good mechanical strength [7-9]. Among REBCO CC tapes, GdBCO CC tape showed better electrical properties under external magnetic fields as compared to those of YBCO or SmBCO CC tapes [10-12].

Evaluation of electro-mechanical properties in coated conductors is important because various kinds of stresses and strains can be induced during manufacturing and operation due to extreme conditions. It was noted that significant degradation of $I_{\mathrm{c}}$ occurred when the applied strain exceeds the irreversible strain limit and cannot be reversibly recovered due to the formation of crack on the superconducting coating film [13-18]. To suppress the initiation of crack, $\mathrm{Cu}$ layer protection is added to the $\mathrm{CC}$ tape thus enhancing the reversibility of $I_{\mathrm{c}}$ and providing further thermal and electrical stability [15-18]. From the previous report of author's group, it was found that different stabilizers such as $\mathrm{Cu}$ or stainless steel provided different extent of enhancement in reversible strain limit of YBCO CC tape [17]. Moreover, a strong substrate material is expected to enhance the axial strain performance and to promote good mechanical support to the $\mathrm{CC}$ tape such as $\mathrm{Ni}$ alloy which is used in RABiTS process and Hastelloy for IBAD process. It is of great concern to fabricate CC tapes with inexpensive substrate having good mechanical properties comparable to commercially available $\mathrm{CC}$ tapes with Hastelloy substrate.

* Corresponding author: hsshin@andong.ac.kr
In this study, the mechanical and electro-mechanical properties of the GdBCO CC tapes adopting stainless steel substrate with additional $\mathrm{Cu}$ stabilizer and brass laminate were measured at $77 \mathrm{~K}$ under self-field and quantitatively analyzed. The thermal contraction and coefficient of thermal expansion (CTE) of CC tapes of each constituent layers were measured and used to determine the residual strain induced in the GdBCO coating film. The results are then compared with that of GdBCO CC tapes with Hastelloy substrate [18].

\section{EXPERIMENTAL PROCEDURE}

\subsection{Samples}

In order to investigate the influence of stabilizing layer on the electro-mechanical property, three kinds of GdBCO $\mathrm{CC}$ samples, Ag-stabilized, $\mathrm{Cu}$-stabilized and Brass laminated CC samples, were supplied by SuNAM Co., Ltd. for this study.

Fig. 1 shows the architecture of RCE-DR GdBCO CC tapes. The coating film was deposited on the stainless steel substrate using reactive co-evaporation by deposition and reaction (RCE-DR) process. Ag layer was sputtered onto the coating film which serves as protection layer. Then, copper was electroplated which surrounds the $\mathrm{CC}$ tape. Additional reinforcement of brass laminates were soldered on both sides of the tape.

The bare stainless steel substrate was also obtained by removing the $\mathrm{Ag}$ and $\mathrm{GdBCO}$ film from $\mathrm{Ag}$-stabilized $\mathrm{CC}$ tape through the etching process.

The specifications of the samples tested were shown in Table I.

\subsection{Measurement of mechanical properties}

Tensile tests of each GdBCO CC tape samples and bare stainless steel substrate were carried out at $77 \mathrm{~K}$ using a 


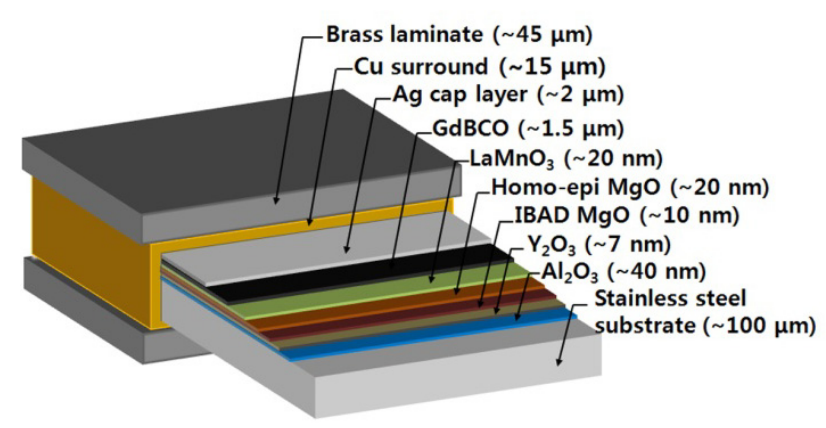

Fig. 1. Architecture of RCE-DR GdBCO CC tape with stainless steel substrate. It shows the component layers and each thickness.

TABLE I

SPECIFICATIONS OF GDBCO CC SAMPLES.

\begin{tabular}{lc}
\hline & $\mathrm{IBAD} / \mathrm{GdBCO} \mathrm{CC}$ \\
\hline Fabrication process & $\mathrm{RCE}-\mathrm{DR}$ \\
Structure & $\mathrm{Ag} / \mathrm{GdBCO} / \mathrm{LaMnO} /$ \\
& $\mathrm{Homo}-\mathrm{epi} \mathrm{MgO} / \mathrm{IBAD} \mathrm{MgO} /$ \\
& $\mathrm{Y}_{2} \mathrm{O}_{3} / \mathrm{Al}_{2} \mathrm{O}_{3} /$ Stainless steel \\
Dimension, $\mathrm{t} \times \mathrm{w}(\mathrm{mm})$ & \\
$\mathrm{Ag}$-stabilized & $0.105 \times 3.98$ \\
Cu-stabilized & $0.139 \times 4.02$ \\
Brass laminated & $0.234 \times 4.19$ \\
Manufacturer & SuNAM \\
\hline
\end{tabular}

universal tensile testing machine (Shimadzu AG-IS, $5 \mathrm{kN}$ load cell) at a constant cross-head speed of $1 \mathrm{~mm} / \mathrm{min}$ [12]. A $15 \mathrm{~mm}$ gauge length Nyilas-type double extensometer was directly attached at the central part of the sample to measure the induced strain during uniaxial tension. The sample gauge length for tensile test was $40 \mathrm{~mm}$. Elastic modulus and yield strength were determined.

The measured elastic moduli of GdBCO CC tapes were then compared with the estimation of elastic modulus using the rule of mixture described by the following equations $[18,19]$ :

$$
\begin{aligned}
& E_{\text {Ag-stab. }}=E_{A g} V_{A g}+E_{S T S} V_{S T S} \\
& E_{\text {Cu-stab. }}=E_{C u} V_{C u}+E_{A g} V_{A g}+E_{S T S} V_{S T S} \\
& E_{\text {Brass lam. }}=E_{\text {Brass }} V_{\text {Brass }}+E_{C u} V_{C u}+E_{A g} V_{A g}+E_{S T S} V_{S T S}
\end{aligned}
$$

where $E_{i}$ and $V_{i}$ correspond to the elastic modulus and volume fraction of each constituent layer $i$, respectively.

The thermal contraction and coefficient of thermal expansion (CTE) of three kinds of GdBCO CC tapes and constituent layers such as stainless steel substrate, $\mathrm{Cu}$ stabilizer, and brass laminate were measured using double extensometer. The measuring procedure is described in detail in [20].

The thermal contraction and CTE of each sample are calculated by the following equations:

$$
\begin{gathered}
\frac{\Delta L}{L}=\frac{\Delta V_{\text {sample }}-V_{\text {ext. }}}{\left(C F_{@ 77 K}\right)\left(G L_{\text {ext } .}\right)} \\
\alpha=\frac{\Delta L / L}{\Delta T}
\end{gathered}
$$

where $\Delta \mathrm{L} / \mathrm{L}$ is the thermal contraction of the sample; $\Delta \mathrm{V}_{\text {sample }}$ corresponds to the difference in voltage reading of the sample in cooling from RT to $77 \mathrm{~K}$; $\mathrm{V}_{\text {ext. }}$ is the initial voltage reading of the double extensometer; $\mathrm{CF}_{@ 77 \mathrm{~K}}$ is the calibration factor at $77 \mathrm{~K}$ which is 1.82 in this study; $\mathrm{GL}_{\text {ext. }}$ is the gauge length of the double extensometer, 15 $\mathrm{mm} ; \alpha$ is the coefficient of thermal expansion (CTE); and $\Delta \mathrm{T}$ represents the temperature difference from $\mathrm{RT}$ to $77 \mathrm{~K}$.

\subsection{Measurement of $I_{\mathrm{c}}$ under uniaxial tension}

The response of $I_{\mathrm{c}}$ depending on uniaxial strain/stress was measured at $77 \mathrm{~K}$ under self-field for the CC sample mounted to a loading frame installed on the universal tensile test machine. Both ends of the sample were fixed by copper grips which serve as electric contacts to current leads. The gauge length of the sample was $40 \mathrm{~mm}$ and voltage taps were soldered at the middle part of the sample with a separation distance of $20 \mathrm{~mm}$. The strain induced to the sample due to the application of tensile load was measured by double extensometer attached between both voltage taps. $I_{\mathrm{c}}$ was measured at a voltage criterion of 1 $\mu \mathrm{V} / \mathrm{cm}$ for every $0.05 \%$ interval of applied uniaxial strain on the sample. When an $I_{\mathrm{c}}$ degradation of about $5 \%$ occurred, the applied tensile load was released to check the reversible behavior of $I_{\mathrm{c}}$ against strain. The sample was loaded and unloaded periodically to determine its irreversible strain and stress limits, $\varepsilon_{\text {irr }}$ and $\sigma_{\text {irr }}$ respectively, which have been defined in this study as $99 \% I_{\mathrm{c}}$ recovery during unloading.

\section{RESULTS AND DISCUSSION}

\subsection{Mechanical properties}

Stress-strain curves of $\mathrm{GdBCO} \mathrm{CC}$ tapes and bare stainless steel substrate obtained at $77 \mathrm{~K}$ are shown in Fig. 2. Elastic modulus was determined from the initial linear slope of each curves and the yield strength was obtained from $0.2 \%$ offset strain. From the results, it can be found that additional $\mathrm{Cu}$ and brass lamination have caused the decrease in the elastic modulus and yield strength of the samples.

As a reference, the results of $\mathrm{GdBCO} \mathrm{CC}$ tapes with stainless steel substrate were compared with the GdBCO CC tapes adopting Hastelloy substrate [18]. As can be observed in Table II, all CC tapes with Hastelloy substrate showed higher mechanical properties than those with stainless steel substrate.

When using Eq. (1) for Ag-stabilized CC tape, it resulted to an estimated $E$ value of $205 \mathrm{GPa}$ which is almost similar with the measured value of $207 \mathrm{GPa}$. The calculated $E$ value of $187 \mathrm{GPa}$ for $\mathrm{Cu}$-stabilized CC tape using Eq. (2) also showed similarity with the measured one of $186 \mathrm{GPa}$. But in the case of Brass laminated using Eq. (3), the calculated $E$ value of $155 \mathrm{GPa}$ showed a small difference from the measured one of $141 \mathrm{GPa}$. This indicates that disregarding the solder layer in the calculation will result to the difference between the measured and calculated $E$ values. 


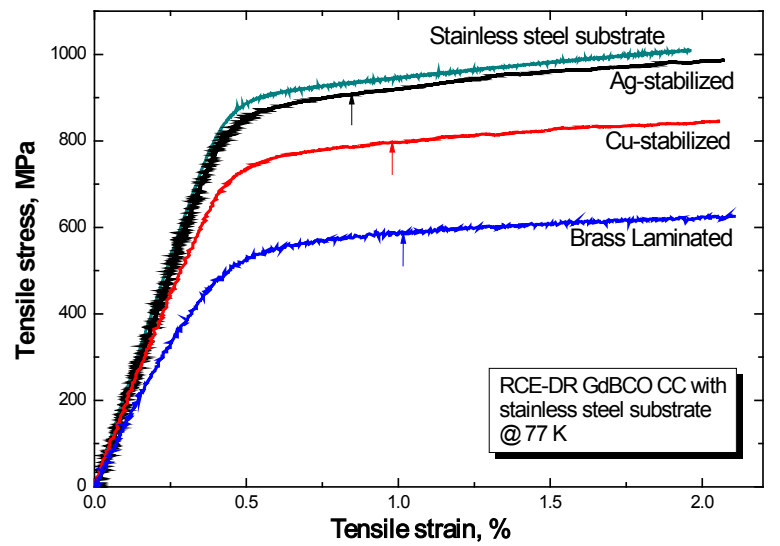

Fig. 2. Stress-strain curves of GdBCO CC samples and bare stainless steel substrate obtained through uniaxial tensile tests.

TABLE II

MECHANICAL PROPERTIES OF GDBCO CC TAPES.

\begin{tabular}{cccc}
\hline & $\begin{array}{c}\text { Elastic } \\
\text { modulus, } \\
E(\mathrm{GPa})\end{array}$ & $\begin{array}{c}\text { Yield } \\
\text { strength, } \\
\sigma_{\mathrm{y}}(\mathrm{MPa})\end{array}$ & $\begin{array}{c}\text { Yield } \\
\text { strain, } \\
\varepsilon_{\mathrm{y}}(\%)\end{array}$ \\
\hline Stainless steel substrate & 212 & 914 & 0.62 \\
$\mathrm{Ag}$-stabilized & 207 & 885 & 0.64 \\
Cu-stabilized & 186 & 764 & 0.61 \\
Brass laminated & 141 & 548 & 0.59 \\
\hline Hastelloy substrate* & 232 & 1285 & 0.73 \\
Ag-stabilized* & 237 & 1244 & 0.75 \\
Cu-stabilized* & 191 & 887 & 0.71 \\
Brass laminated* & 148 & 626 & 0.65 \\
\hline
\end{tabular}

$$
\text { *[18] }
$$

\section{2. $I_{\mathrm{c}}$-strain response of $\mathrm{GdBCO} \mathrm{CC}$ tapes under uniaxial} tension

Fig. 3 shows the response of $I_{\mathrm{c}}$ under the application of uniaxial tensile strain/stress in GdBCO CC tapes with stainless steel substrate. From Fig. 3(a), Ag-stabilized sample showed less strain sensitivity of $I_{\mathrm{c}}$ at lower strain values than the other $\mathrm{CC}$ samples. However, when the strain was further increased over $0.80 \%$, Ag-stabilized sample showed sudden $I_{\mathrm{c}}$ degradation to about $30 \%$ of the original $I_{\mathrm{c}}$ value. On the other hand, $\mathrm{Cu}$-stabilized and Brass laminated samples showed continuous gradual $I_{\mathrm{c}}$ degradation behavior up to higher strain values. In addition, both samples showed almost similar $I_{\mathrm{c}}$ degradation behavior and had higher irreversible strain limits of $0.95 \%$ and $1.0 \%$, respectively. This behavior for both $\mathrm{Cu}$-stabilized and Brass laminated samples may be attributed to similar thermal contraction between major constituent layers resulting in an even pre-compression strain induced to the GdBCO coating film which will be discussed more in the following subsection.

Although the Cu-stabilized and Brass laminated samples showed higher tolerant strain limit, both have lower irreversible stress limits of $775 \mathrm{MPa}$ and $568 \mathrm{MPa}$, respectively, than that of the Ag-stabilized sample with $872 \mathrm{MPa}$. This resulted from the additional copper and brass layers which decreased the apparent elastic modulus and yield strength of the whole CC tapes.
The $\varepsilon_{\text {irr }}$ and $\sigma_{\text {irr }}$ of GdBCO CC tapes with stainless steel substrate and those of Hastelloy substrate are shown in Table III. It can be apparently observed that samples with stainless steel substrate showed much higher $\varepsilon_{\text {irr }}$, but have lower $\sigma_{\text {irr }}$ than that of samples with Hastelloy substrate. The Ag-stabilized, Cu-stabilized, and Brass laminated samples with stainless steel substrate have $\varepsilon_{\text {irr }}$ differences of $0.35 \%, 0.40 \%$, and $0.25-0.30 \%$, respectively, from the samples adopting Hastelloy substrate. These only show that GdBCO CC tapes with stainless steel substrate are more strain tolerant than those with Hastelloy substrate.

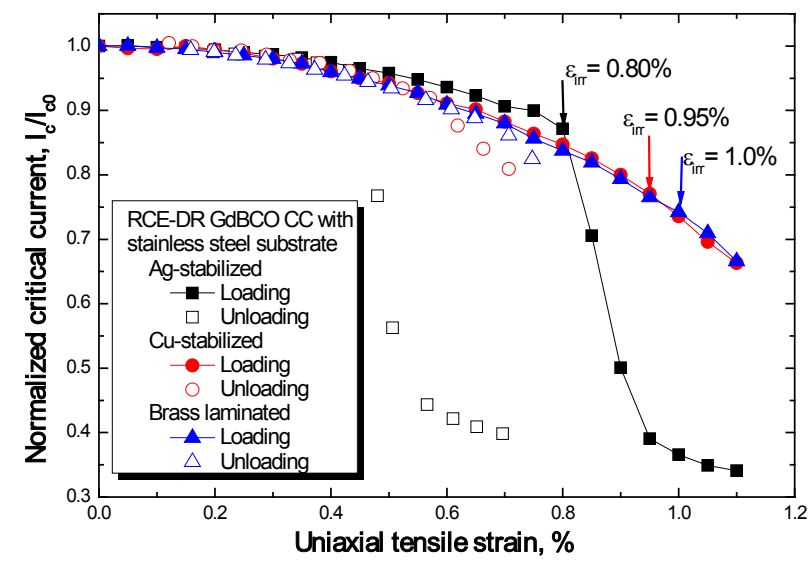

(a)

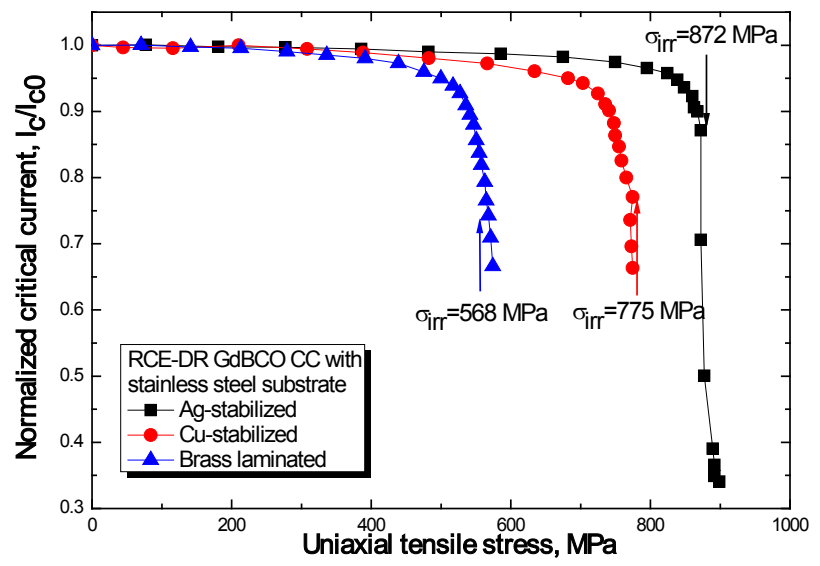

(b)

Fig. 3. Degradation behavior of normalized critical current with uniaxial (a) tensile strain and (b) tensile stress in GdBCO CC tapes with stainless substrate at $77 \mathrm{~K}$ under self-field.

TABLE III

IRREVERSIBLE STRAIN AND STRESS LIMITS OF GDBCO DD TAPES.

\begin{tabular}{ccccc}
\hline \multirow{2}{*}{$\begin{array}{c}\text { GdBCO CC } \\
\text { samples }\end{array}$} & \multicolumn{2}{c}{$\begin{array}{c}\text { With stainless steel } \\
\text { substrate }\end{array}$} & \multicolumn{2}{c}{$\begin{array}{c}\text { With Hastelloy } \\
\text { substrate* }\end{array}$} \\
& $\varepsilon_{\text {irr }}(\%)$ & $\boldsymbol{\sigma}_{\text {irr }}(\mathrm{MPa})$ & $\varepsilon_{\text {irr }}(\%)$ & $\boldsymbol{\sigma}_{\text {irr }}(\mathrm{MPa})$ \\
\hline Ag-stabilized & 0.80 & 872 & 0.45 & 1,035 \\
Cu-stabilized & 0.95 & 775 & 0.55 & 800 \\
Brass laminated & 1.0 & 568 & $0.70-0.75$ & 600 \\
\hline$*[18]$ & & & &
\end{tabular}


As shown in Fig. 2, the irreversible strain limits of each GdBCO CC sample with stainless steel substrate were indicated as arrows and were located in the plastic region on the stress-strain curves. But, in the case of GdBCO CC tapes with Hastelloy substrate, the irreversible strain of Ag-stabilized sample was located in the elastic region before the onset of yielding [18].

3.3. Strain induced on the GdBCO coating film by the constituent layers

During cool down from RT to $77 \mathrm{~K}$, the GdBCO coating film experiences additional pre-compression strain due to difference in the CTE among the constituent layers of each composite tape. In order to determine the additional pre-compression strain induced to the coating film, therefore, it is necessary to analyze the thermal contraction and CTE of each CC tape and its major constituent layers

Table IV shows the thermal contraction $(\Delta \mathrm{L} / \mathrm{L})$ and $\mathrm{CTE}$ of GdBCO $\mathrm{CC}$ tapes with stainless steel substrate and major constituent layers including the substrate, copper, and brass laminate. These values could be calculated using Eqs. (4) and (5). In the case of CC tapes with stainless steel substrate, it can be observed that the major constituent layers have almost similar thermal contraction that may be the reason of similar $I_{c}$ degradation behavior of $\mathrm{Cu}$-stabilized and Brass laminated samples.

To calculate the pre-compressive strain induced by these constituent layers on the GdBCO film, $\varepsilon_{\mathrm{GdBCO}}$, after cooling from RT to $77 \mathrm{~K}$, the following equation was used [15],

$$
\varepsilon_{G d B C O}=\left(\frac{\Delta L}{L}\right)_{G d B C O}-\left(\frac{\Delta L}{L}\right)_{C C}
$$

where $(\Delta L / L)_{G d B C O}$ is the thermal contraction of $\mathrm{GdBCO}$ film which is assumed as $0.14 \%$, same as average thermal contraction of $\mathrm{YBCO}[7]$; and $(\Delta L / L)_{C C}$ is the thermal contraction of the whole CC tape.

Using both Eq. (6) and thermal contractions of CC tapes from Table IV, the pre-strain values induced on the GdBCO film for Ag-stabilized, $\mathrm{Cu}$-stabilized and Brass laminated $\mathrm{CC}$ tapes were calculated and they are $0.10 \%$, $0.15 \%$, and $0.16 \%$, respectively. Almost similar pre-compression strain was induced to the GdBCO coating film of the $\mathrm{Cu}$-stabilized and Brass laminated samples. Therefore, both samples have shown similar strain response of $I_{\mathrm{c}}$ and irreversible strain limits as demonstrated in Fig. 3(a).

TABLE IV

THERMAL CONTRACTION AND CTE MEASURED FOR GDBCO CC TAPES AND CONSTITUENT LAYERS

\begin{tabular}{lcc}
\hline GdBCO CC tapes & $\Delta \mathrm{L} / \mathrm{L}(\%)$ & $\mathrm{CTE}\left(\mathbf{1 0}^{-6} \mathrm{~K}^{-1}\right)$ \\
\hline Ag-stabilized & $\mathbf{- 0 . 2 4} \pm \mathbf{0 . 0 3}$ & $\mathbf{1 1 . 2 5} \pm \mathbf{1 . 5 1}$ \\
Cu-stabilized & $\mathbf{- 0 . 2 9} \pm \mathbf{0 . 0 4}$ & $13.17 \pm 0.91$ \\
Brass laminated & $-\mathbf{0 . 3 0} \pm \mathbf{0 . 0 5}$ & $13.97 \pm 2.85$ \\
\hline Constituent layers & & \\
Stainless steel substrate & $-\mathbf{0 . 3 2} \pm \mathbf{0 . 0 5}$ & $\mathbf{1 4 . 5 8} \pm \mathbf{2 . 2 9}$ \\
Copper & $\mathbf{- 0 . 3 3} \pm \mathbf{0 . 0 1}$ & $\mathbf{1 5 . 2 1} \pm \mathbf{0 . 5 9}$ \\
Brass laminate & $\mathbf{- 0 . 3 4} \pm \mathbf{0 . 0 1}$ & $\mathbf{1 5 . 6 2} \pm \mathbf{0 . 4 8}$ \\
\hline
\end{tabular}

\section{CONCLUSIONS}

The mechanical and electro-mechanical properties of GdBCO CC tapes with stainless steel substrate were investigated through experimental measurement and analytical calculation. Results showed that these tapes have higher strain tolerance limits and have comparable mechanical strength to those $\mathrm{GdBCO} \mathrm{CC}$ tapes with Hastelloy substrate. Improvement in irreversible strain limit was attained from the additional copper and brass layers to the $\mathrm{CC}$ tapes. Moreover, similar thermal contraction and CTE values between the layers of substrate and stabilizer materials have brought similar strain tolerance due to almost similar pre-compression strain induced to the superconducting film.

\section{ACKNOWLEDGMENT}

This work was supported by the National Research Foundation of Korea (NRF) funded by the Korean government (MEST) (2011-0015369).

\section{REFERENCES}

[1] M. Ohya, Y. Ashibe, M. Watanabe, T. Minamino, H. Yumura, T Masuda, and T. Kato, "Development of RE-123 superconducting cable," IEEE Trans. Appl. Supercond., vol. 19, no. 3, pp. 176-1769, 2009.

[2] M. Iwakuma, Y. Hase, T. Satou, A. Tamioka, M. Konno, Y. Iijima, T. Saitoh, Y. Yamada, T. Izumi, and Y. Shiohara, "Production and Test of a REBCO Superconducting Synchronous Motor," IEEE Trans. Appl. Supercond., vol. 19, no. 3, pp. 1648-51, 2009.

[3] D. W. Hazelton, V. Selvamanickam, J. M. Duval, D. C. Larbalestier, W. D. MArkiewicz, H. W. Weijers, and R. L. Holtz, "Recent Developments in 2G HTS Coil Technology," IEEE Trans. Appl. Supercond., vol. 19, no. 3, pp. 2218-22, 2009.

[4] K. Watanabe, S. Awaji, G. Nishijima, T. Kiyoshi, H. Kumakura, S. Hanai, K. Koyanagi, and M. Ono, "20 T compact superconducting outsert employing Y123 coated conductors for a $45 \mathrm{~T}$ hybrid magnet," IEEE Trans. Appl. Supercond., vol. 19, no. 3, pp. 1592-95, 2009.

[5] J. K. Lee, S. H. Park, Y. Kim, S. Lee, H. G. Joo, W. S. Kim, K. Choi, and S. Y. Hahn, "Test results of a $5 \mathrm{~kW}$ fully superconducting homopolar motor," Prog .in Supercond. and Cryo., vol. 15, no. 1, pp. 35-39, 2013

[6] J. W. Ekin, "Experimental Techniques for Low-temperature Measurements," Oxford University Press Inc., New York, 2006.

[7] D. C. van der Laan, and J. W. Ekin, "Large intrinsic effect of axial strain on the critical current of high-temperature superconductors for electric power applications," Appl. Phys. Lett., vol. 90, pp. 052506, 2007.

[8] M. Sugano, T. Nakamura, T. Manabe, K. Shikimachi, N. Hirano, and S. Nagaya, "The intrinsic strain effect on critical current under a magnetic field parallel to the c-axis for a MOCVD-YBCO coated conductor," Supercond. Sci. Technol., vol. 21, no. 11, pp. 115019 , 2008.

[9] H. S. Shin, and M. J. Dedicatoria, "Variation of the strain effect on the critical current due to external lamination in REBCO coated conductors," Supercond. Sci. Technol., vol. 25, pp. 054013, 2012.

[10] A. Kinoshita, K. Takahashi, H. Kobayashi, Y. Yamada, A. Ibi, H. Fukushima, M. Konishi, S. Miyata, Y. Shiohara, T. Kato, and T. Hirayama, "Investigation of a thick PLD-GdBCO and $\mathrm{ZrO}_{2}$ doped GdBCO coated conductors with high critical current on $\mathrm{PLD}-\mathrm{CeO}_{2}$ capped IBAD-GZO substrate tapes," Supercond. Sci. Technol., vol. 19, pp. 924, 2006.

[11] R. Fuger, M. Inoue, K. Higashikawa, T. Kiss, M. Namba, S. Awaji, K. Watanabe, A. Ibi, H. Fukushima, Y. Yamada, and T. Izumi, 
"Evaluation of current transport properties of $\mathrm{GdBa}_{2} \mathrm{Cu}_{3} \mathrm{O}_{7-\delta}$ coated conductors over a wide range of temperature and external magnetic fields," J. Phys.: Conf. Ser., vol. 234, pp. 022009, 2010.

[12] M. J. Dedicatoria, H. S. Shin, H. S. Ha, S. S. Oh, and S. H. Moon, "Electro-mechanical property evaluation of REBCO coated conductor tape with stainless steel substrate," Supercond. Cryo., vol. 12, no. 4, pp. 20-23, 2010

[13] N. Cheggour, J. W. Ekin, C. C. Clickner, D. T. Verebelyi, C. L. H. Thieme, R. Feenstra, and A. Goyal, "Reversible axial-strain effect and extended strain limits in $\mathrm{Y}-\mathrm{Ba}-\mathrm{Cu}-\mathrm{O}$ coatings on deformation-textured substrates," Appl. Phys. Lett., vol. 83, pp 4223-5, 2003

[14] M. Sugano, K. Osamura, W. Prusseit, R. Semerad, T. Kuroda, K. Itoh, and T. Kiyoshi, "Irreversible strain dependence of critical current in 100 A class coated conductors," IEEE Trans. Appl. Supercond., vol. 15, no. 2, pp. 3581-84, 2005.

[15] N. Cheggour, J. W. Ekin, Y. Y. Xie, V. Selvamanickam, C. L. Thieme, and D. T. Verebelyi, "Enhancement of the irreversible axial-strain limit of $\mathrm{Y}-\mathrm{Ba}-\mathrm{Cu}-\mathrm{O}$ coated conductors with the addition of a Cu layer," Appl. Phys. Lett., vol. 87, pp. 212505, 2005.
[16] N. Cheggour, J. W. Ekin, C. L. H. Thieme, Y. Y. Xie, V. Selvamanickam, and R. Feenstra, "Reversible axial-strain effect in Y-Ba-Cu-O coated conductors," Supercond. Sci. Technol., vol. 18, pp. S319-S324, 2005.

[17] H. S. Shin, K. H. Kim, J. R. C. Dizon, T. Y. Kim, R. K. Ko, and S. S. $\mathrm{Oh}$, "The strain effect on critical current in YBCO coated conductors with different stabilizing layers," Supercond. Sci. Technol., vol. 18, pp. S364-S368, 2005.

[18] M. J. Dedicatoria, and H. S. Shin, "Analysis on stress/strain tolerances of $I_{c}$ in externally laminated GdBCO CC tapes," IEEE Trans. Appl. Supercond., vol. 23, no. 3, pp. 8400504, 2013.

[19] K. Osamura, S. Machiya, Y. Tsuchiya, and H. Suzuki, "Internal strain and mechanical properties at low temperatures of surround $\mathrm{Cu}$ stabilized YBCO coated conductor," IEEE Trans. Appl. Supercond. vol. 20, pp. 1532-1536, 2010

[20] M. J. Dedicatoria, J. R. C. Dizon, H. S. Shin, and K. D. Sim, "Establishment of CTE measurement procedure for PPLP at $77 \mathrm{~K}$ for HTS power cables using double extensometers," Supercond. and Cryo., vol. 14, no. 4, pp. 24-27, 2012. 